\title{
LQHR: A Link Quality-based Hybrid Routing Protocol for Wireless Ad Hoc Networks
}

\author{
Ken Nakaoka ${ }^{\dagger}$ \\ tDigital Systems Research Center, \\ SANYO Electric Co., Ltd. \\ 180, Ohmori, Anpachi-cho, Anpachi-gun, \\ Gifu 503-0195 \\ J apan \\ nakaoka@gf.hm.rd.sanyo.co.jp
}

Makoto Obatt

\author{
${ }^{+}$Graduate School of Engineering, \\ Nagoya Institute of Technol ogy \\ Gokiso-cho, Showa-ku, Nagoya, \\ Aichi 466-8555 \\ J apan \\ \{oba, tasaka\}@inl.elcom.nitech.ac.jp
}

\begin{abstract}
This paper proposes a Link Quality-based Hybrid Routing $(L Q H R)$ protocol for wireless ad hoc networks. LQHR takes account of link quality such as SNR between adjacent nodes and the link utilization level of each node. In LQHR, each node maintains routing information produced by OLSR, which is a proactive routing protocol for ad hoc networks. When a source makes a communication request, it finds a route to the destination node on the basis of the link quality. We implemented LQHR on an experimental network, where laptop PCs were arranged on the same floor of a building, and audio-video streams were transmitted along a route selected by LQHR. To evaluate the effectiveness of LQHR in audiovideo transmission, we compared application-level QoS of LQHR with that of the standard OLSR in the presence of another traffic flow in the network. As a result, we found that the routes selected by LQHR can provide shorter transmission delays and lower loss ratios in the transmission of the audiovideo streams than those selected by the standard OLSR.
\end{abstract}

\section{INTRODUCTION}

Research issues regarding ad hoc networks, which attract many researchers' attention, are mainly concerned with routing protocols. A large number of routing protocols for ad hoc networks have been proposed so far. Some of them have been investigated in the MANET working group [1], which aims at standardizing IP routing protocols. MANET is currently developing two routing protocol specifications: Reactive MANET Protocol (RMP) and Proactive MANET Protocol $(P M P)$. RMP and PMP are based on the on-demand protocols and the table-driven ones, respectively; these two kinds of protocols have been discussed in MANET. In RMP, a source node wishing to send a packet initiates a route discovery procedure that finds a route to the destination node. In PMP, on the other hand, each node maintains routing information on all other nodes in the network by exchanging control packets with neighbouring nodes.

Most of the existing protocols, as well as RMP and PMP, employ a hop count to the destination node as the routing metric and select the shortest route, i.e., the minimum hop count route. These shortest route algorithms can provide short delays because of decrease in the number of packet forwarding. As a result, however, each wireless link on the route tends to be longer in geographical distance. This leads to a reduction of packet signal level which is being received.

In ad hoc networks, the short range wireless communication devices such as IEEE 802.11a, 11b and 11g [2] are widely employed. The IEEE 802.11b Wireless LAN standard [3], for instance, supports four data rates: 1, 2, 5.5 and 11 Mbps. The data rates use different modulation schemes and are controlled in accordance with a wireless link quality. On the whole, as the distance of the link becomes longer, the Signalto-Noise Ratio (SNR) degrades; as a consequence, the data rate is switched to a lower one, which then results in lower end-to-end throughput.

A long range link with lower SNR also causes a higher ratio of packet loss. If a routing control packet such as a hello packet collapses on a link, the link is assumed to be broken, and then a route rediscovery procedure is invoked to find a new route. A link failure brings about successive packet loss unless an intermediate node keeps the received packets until a new route is discovered. A route which is selected by the shortest path algorithm is likely to consist of long and unreliable links, and thus packet flows often suffer severe performance degradation [4].

A control message packet such as a route request packet and a hello packet, which is used for route discovery and maintenance, is transmitted as a broadcast packet. In the IEEE 802.11b, for example, the broadcast packet is transmitted with a data rate of 1 or 2 Mbps. On the other hand, a data packet is transmitted as a unicast packet, which is transmitted with a data rate up to $11 \mathrm{Mbps}$. As a rule, as the data rate gets higher, the distance in which a packet can travel without error becomes shorter. This often brings about a problem called communication gray zones; a data packet which is sent by a node with a higher data rate cannot be received by another node even if there is a route between the two nodes [5], [6].

We can find several papers that study the problems of the shortest route and communication gray zones [7]-[11]. Awerbuch et al. [7] proposed Medium Time Metric (MTM) for multi-rate ad hoc networks. MTM assigns each link a weight that is proportional to reciprocal of the data rate; it offers shorter and more reliable links with higher throughput. Couto et al. [8] evaluated the performance of minimum hop count routing on a wireless testbed and proposed the Expected Transmission Count (ETX) metric, which finds highthroughput paths based on packet loss ratios in both directions of each wireless link. In [9], Dube et al. proposes Signal Stability based Adaptive Routing (SSA), which utilizes the signal strength and location stability so as to select longer-lived and stable routes. Itaya et al. [10] introduced Fisheye State Routing with Signal Strength (FSR-SS), which avoids using an unstable link by ignoring hello packets with lower signal lev- 
els than a pre-defined threshold value. Watanabe et al. [11] studied FSR with Metric due to RSSI (FSR-MS), which utilizes a value proportional to reciprocal of the signal strength. They also showed that a route which is one hop or two hops longer than the shortest one can provides fewer packet loss and higher stability.

In addition to considering the received signal level, which can be regarded as reliability of the wireless link, a route should be selected by reflecting the existence of other traffic flows in the network. Lee et al. [13] proposed the Dynamic Load-Aware Routing (DLAR) protocol, which uses the number of packets buffered in the queue of each node as the routing metric. In [14], Takahashi et al. examine Busy Node Avoidance Routing (BNAR) for load balancing in ad hoc networks. These proposed protocols take account of the link utilization level and the load of intermediate nodes, but they do not consider any metric of the link reliability such as the received signal level.

In this paper, we propose a routing protocol which takes account of link quality representing both reliability and the link utilization level of each node; we refer to this protocol as Link Quality-based Hybrid Routing (LQHR). In LQHR, each node maintains routing information produced by an existing proactive routing protocol and measures link quality between the neighbouring nodes. When a source node makes a communication request, it selects a route to the destination node by referring to the link quality on an on-demand basis. LQHR adopts Optimized Link State Routing (OLSR) as the proactive routing protocol, which has been specified in MANET and submitted as Experimental RFC [12].

When using an IEEE 802.11b wireless LAN device, a data packet is transmitted with either of the four data rates as mentioned earlier. In LQHR, link quality such as the signal level, SNR, and the loss ratio of received packets is measured at each data rate. Taking account of the link quality other than the received signal level [10], [11], LQHR can select not only reliable routes but also the routes that meet the requirement of each traffic flow, for example, high throughput or low delay. Moreover, LQHR also employs the link utilization level of each node as link quality in order to reflect the network load in route selection.

We have implemented LQHR on an experimental system, where laptop PCs each equipped with an IEEE 802.11b PC card were arranged on the same floor of a building. To assess the effectiveness of LQHR, we compared the performance of LQHR with that of the standard OLSR under the condition that there exists another traffic flow in the network. In particular, we confirmed the quality-enhancive feature of LQHR by conducting an experiment on audio-video transmission, which requires a reliable route with high throughput.

In Quality of Service (QoS) assessment of audio-video transmission, we must consider the application-level QoS since audio-video information has the temporal structure as well as the spatial structure. Note that QoS parameters such as packet delivery ratio and throughput, which are commonly used in the study on ad hoc networks, do not reflect these information structures. Thus, this paper assesses applicationlevel QoS of audio-video transmission. We refer to the transmission unit at the application-level as a Media Unit (MU); in this paper, we define a video frame as a video MU and a constant number of audio samples as an audio MU.

The rest of this paper is organized as follows. Section II proposes LQHR, and Section III describes the characteristics of LQHR. Section IV explains the experimental methodology, and Section V shows and discusses the results of the experiment. Section VI concludes the paper.

\section{LQHR}

LQHR is a hybrid protocol that consists of two modules:

- Quality Measurement (QM) Module

The QM module produces and maintains routing information by means of a proactive routing protocol. It also periodically measures the link quality between adjacent nodes. The link quality is represented as a vector.

\section{- Route Selection (RS) Module}

The RS module selects a route to the destination node by referring to the link quality, which is measured by the QM module, on an on-demand basis when a communication request is made at a node.

\section{A. Quality Measurement Module}

The QM module uses an existing proactive routing protocol to produce and maintain routing information. In addition to the route maintenance, each node in the network periodically transmits a probe packet to adjacent nodes in order to know the link quality between the two adjacent nodes. A constant number of probe packets are transmitted in a period at each data rate, i.e., each of 1, 2, 5.5 and $11 \mathrm{Mbps}$ in the case of IEEE $802.11 \mathrm{~b}$. The adjacent nodes count the number of probe packets which has been received successfully at each data rate. Whenever receiving the probe packets, the adjacent nodes measure the signal level and SNR of each packet and then take an average of the signal level and that of SNR at each data rate. Thus, the number of received packets, the average value of signal level and that of SNR at each data rate become components of the link quality vector. The information on the link quality vector is sent to the node which transmitted the probe packets. The link quality information is sent either after the measurement at each data rate is completed or after the measurement at all the data rates is completed. After sending back the link quality information, the adjacent nodes clear the measurement results and then start the next measurement.

By sending probe packets and then receiving measurement results at each data rate, every node in the network can learn the link quality between the adjacent nodes. The link quality at each data rate is utilized when the node sends a data packet.

In addition to the signal level and SNR, LQHR adopts the link utilization level as the link quality. When IEEE 802.11b is used, the link utilization level for a link is defined as

$$
\text { Utilize }=100 \times\left(\frac{T_{1}}{1}+\frac{T_{2}}{2}+\frac{T_{5.5}}{5.5}+\frac{T_{11}}{11}\right)
$$

where Utilize denotes the link utilization level for the link, $T_{R}$ is the number of bits transmitted or received on the link in a second at a data rate of $R$ Mbps $(\mathrm{R}=1,2,5.5,11)$. As Utilize 


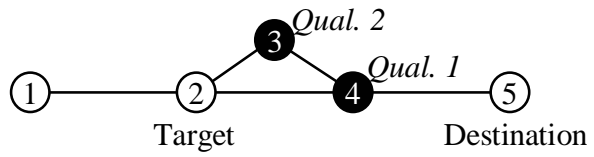

Figure 1: An example of possible next-hop nodes.

becomes smaller, the link utilization level gets lower.

In this paper, LQHR adopts OLSR [12] as the proactive routing protocol. This is because each node needs to know how other nodes are connected to one another in the RS module, which is described later. OLSR is a link state routing protocol; thus each node can grasp the whole network topology. Moreover, since OLSR has been studied in MANET [1] and specified as Experimental RFC [12], it is expected to be widely used as a standard protocol.

\section{B. Route Selection Module}

The RS module selects a route to the destination node by referring to the link quality when a communication request is made at a node. A route is selected on demand since the RS module is invoked before data transmission.

When a communication request is made at the source node, it sends a request message to an adjacent node which satisfies a certain qualification; we refer to the adjacent node as a possible next-hop node in the rest of the paper. After the request message is relayed up to a last-hop node, which is located one hop away from the destination, then a response message is forwarded back to the source node on the route which is selected on the basis of the link quality of each forwarding node. The route thus selected is also used for the transmission of data packets.

In the following, we give the definition of the possible nexthop node and describe how a route from the source to the destination is selected by relaying the request message and the corresponding response message.

\section{1) Definition of Possible Next-hop Node}

A possible next-hop node for a node (say the target node) is defined as a node which has either of the following two qualifications:

\section{Qualification 1}

A node which is selected by OLSR as the next hop node to the destination node; we simply refer to this node as the next-hop node of the target node.

\section{Qualification 2}

A node which is adjacent to the target node and at the same time one hop away from the next-hop node defined in qualification 1.

Figure 1 describes how a possible next-hop node of node 2 is specified; black nodes represent possible next-hop nodes. In Figure 1, node 2 is the target node, node 5 is the destination, and nodes 1, 3 and 4 are adjacent nodes of node 2. Assume that node 4 is selected as the next hop node of node 2 by OLSR in the QM module; therefore, qualification 1 specifies node 4 as a possible next-hop node. Moreover, node 3 is selected as a possible next-hop node with qualification 2 since node 3 is adjacent to the target node and is one hop away from the next-hop node (namely, node 4).

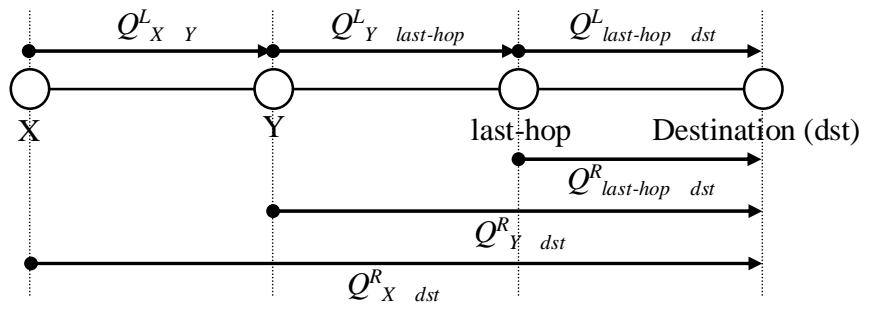

Figure 2: Link quality and route quality between nodes.

A route including possible next-hop nodes with qualification 2 has more hops than the shortest route; in Figure 1, route "2-3-4-5" is one hop longer than route " $2-4-5$ ". Note that it is often the case that a shorter range link is more stable than a longer range one.

\section{2) Route Selection Mechanism}

On having a communication request, the source node sends a Route Quality Request (RQReq) message to each of the possible next-hop nodes. The RQReq message contains the address of the destination node. The nodes receiving the RQReq message refer to the destination address and then forward it to each of their own possible next-hop nodes. The RQReq message is forwarded up to a last-hop node. The last-hop node may receive more than one RQReq message since the node can be a possible next-hop for more than one up-stream node. It is also possible that there exists more than one last-hop node as well. Once the RQReq message reaches the last-hop node, it forwards back a Route Quality Response (RQRsp) message, via the series of the possible next-hop nodes the RQReq message has gone through, finally to the source node; thus a route from the source to the destination is selected. The RQRsp messages are chosen and discarded on the way to the source node on the basis of the link quality of each forwarding node.

We now explain how the RQRsp message is forwarded from the last-hop node to the source node in further detail. Let us define a route quality, which indicates the quality of a route toward the destination node. The route quality is a vector whose components are the same as those of the link quality vector. Figure 2 shows an example of link quality and route quality between nodes. In this figure, the link quality and the route quality of node A toward node $B$, for instance, are represented by $Q_{A \rightarrow B}^{L}$ and $Q_{A \rightarrow B}^{R}$, respectively. Each component in the route quality is defined as the lowest quality of the corresponding component of the link quality among all toward the destination. On receiving the RQReq message, the lasthop node determines the route quality by setting its own link quality $\left(Q_{\text {last-hop } \rightarrow \text { dest }}^{L}\right)$, which is the quality between the lasthop node and the destination node, to the route quality $\left(Q_{\text {last-hop } \rightarrow \text { dest }}^{R}\right.$ ). The last-hop node then sends an RQRsp message, which contains the route quality $\left(Q_{\text {last-hop } \rightarrow \text { dest }}^{R}\right)$, to node $\mathrm{Y}$, which has sent the RQReq message to the last-hop node. On receiving the RQRsp message from the last-hop node, node Y compares its own link quality ( $Q_{Y \rightarrow \text { last-hop }}^{L}$ ), which is the quality between node $\mathrm{Y}$ and the last-hop node, with the route quality ( $Q_{\text {last-hop } \rightarrow \text { dest }}^{R}$ ) included in the RQRsp message received and calculates a new route quality $\left(Q_{Y \rightarrow \text { dest }}^{R}\right)$. The 


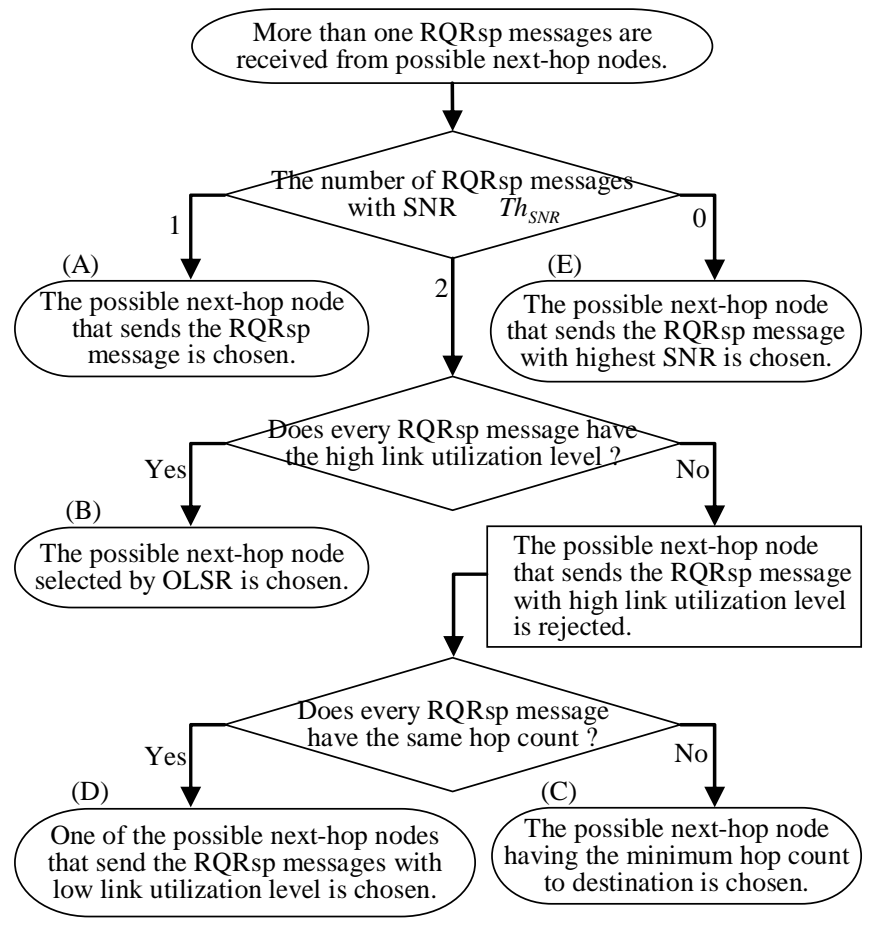

Figure 3: A flow diagram explaining how to choose the next hop node among several possible next-hop nodes.

value of the new route quality component in the RQRsp message is set to the lower quality; we set SNR and the link utilization level, for instance, to the lower value and higher one, respectively. Node Y thus replaces the existing route quality values with the new ones and forwards the RQRsp message to node $\mathrm{X}$, which has sent the RQReq message to node $\mathrm{Y}$. In the same way, the RQRsp message is forwarded back until it reaches the source node.

When a node has one possible next-hop node, the node receives only one RQRsp message; in this case, it inevitably regards the possible next-hop node as the next hop node to the destination.

On the other hand, when a node has more than one possible next-hop node, the node receives the same number of RQRsp messages as that of the possible next-hop nodes. Consequently, when receiving more than one RQRsp message, the node needs to choose one possible next-hop node as the next hop node to the destination on the basis of the route quality, which is included in the received RQRsp messages.

In the above case, the next hop node to the destination is chosen as follows. The flow diagram in Figure 3 explains how to choose the next hop node from among several possible next-hop nodes. Assume that SNR and the link utilization level are employed as the link quality. Each node in the network is given a threshold value $T h_{S N R}$ for SNR. If there is a single RQRsp message whose SNR value in the route quality is equal to or more than the threshold $T h_{S N R}$, then the possible next-hop node that sends the RQRsp message is chosen as the next hop node to the destination; this corresponds to (A) in Figure 3 .

If there is more than one RQRsp message whose SNR value in the route quality is equal to or more than $T h_{S N R}$, a possible next-hop node is chosen by referring to the link utilization level in the route quality. First of all, a node receiving the RQRsp messages classifies the link utilization level in the route quality into three levels, according to threshold values specified in advance, low, middle and high. When all the RQRsp messages have the high link utilization level, a possible next-hop node which is selected by OLSR is chosen as the next hop node to the destination (B). On the other hand, if there are the RQRsp messages with high, middle and low, a possible next-hop node that sends the RQRsp message with high is firstly rejected. From among the rest of the possible next-hop nodes, a possible next-hop node that has the minimum hop count to the destination is chosen (C). If all the nodes have the same hop count, one of the possible next-hop nodes that send the RQRsp messages with low is chosen (D).

If there is no RQRsp message whose SNR is equal to or more than the threshold $T h_{S N R}$, then a possible next-hop node that sends the RQRsp message with highest SNR is chosen (E).

\section{3) Route Re-Selection Mechanism}

Because an ad hoc network can have mobile nodes, the migration of the nodes causes frequent changes of the network topology as well as the fluctuations of the link quality. In order to cope with such a network situation, a source node periodically selects an up-to-date route by sending an RQReq message at regular intervals. A new route is selected in the same way as described earlier.

\section{Characteristics OF LQHR}

A Significant feature of LQHR is that it employs OLSR, which is a proactive routing protocol, though it selects a route on an on-demand basis. That is, LQHR is a hybrid routing protocol in the sense that it is a combination of proactive and reactive routing protocols.

Let us explain the advantages of LQHR. LQHR refers to the link quality in a short period of time by exchanging RQReq and RQRsp messages; therefore, LQHR does not need to distribute the link quality information all over the network unlike proactive routing protocols. Because LQHR employs OLSR as the proactive protocol, the link quality measured by the QM module could be distributed all over the network by piggybacking it onto a routing control packet. In OLSR, we can use a control packet such as a HELLO message or a Topology Control (TC) message. However, it would take some time to distribute the link quality information to the whole network with such control packets. Moreover, mobile nodes cause frequent changes in the link quality. Distributing the link quality information in accordance with changes in network conditions would lead to an increase in the network load.

Although LQHR selects a route on demand, an RQReq message, which is a unicast packet, is transmitted to possible next-hop nodes in a limited area. This is because LQHR has the knowledge of the network topology, which is maintained by OLSR. Note that in a reactive protocol such as AODV and DSR, a route request message is distributed as a broadcast packet on a flooding basis, and then the network traffic increases whenever a route request is made. 


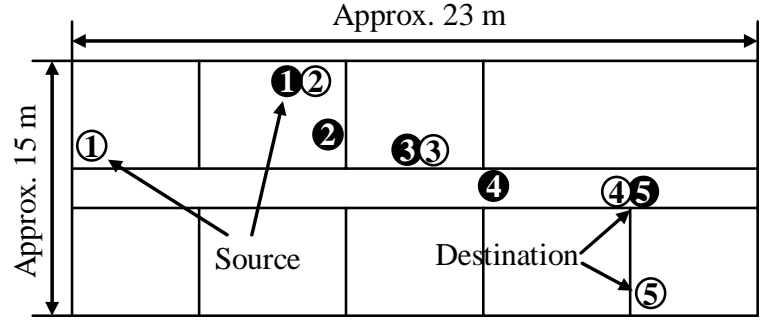

Figure 4: Arrangement of PCs in Experiment I.

Another advantage of LQHR is compatibility with OLSR. LQHR can be considered as a routing protocol which introduces two functions into OLSR: "measurement of the link quality" and "route selection based on the link quality". This allows us to use LQHR in the following way. A communication application initiates the RS module as the need arises; otherwise, only the routing information maintained by OLSR is utilized. Moreover, it is not necessary to modify the existing functions of OLSR when introducing the abovementioned two functions into OLSR; therefore, nodes with LQHR and nodes with OLSR can coexist in the same network.

\section{EXPERIMENTAL METHODOLOGY}

This section describes the configuration of the experimental system for the QoS assessment of LQHR, experimental conditions and QoS parameters used in the experiment. In this paper, we conducted two types of experiments: experiment I and experiment II.

In experiment I, the fundamental performance of LQHR was measured under the condition that there exists no other traffic flow in the network; therefore, LQHR in experiment I does not consider the link utilization level, and we refer to this as $L Q H R$ without Link Utilization $(L U)$. In order to assess the effectiveness of LQHR, we compared LQHR without LU to the standard OLSR.

In experiment II, we examined the effectiveness of LQHR under the condition that there is another traffic flow in the network. We employed two types of LQHR. One is LQHR without LU. The other is the proposed LQHR, which uses the link utilization level as a quality measure as well as SNR; we refer to this as $L Q H R$ with $L U$. We compared LQHR with LU to the standard OLSR and LQHR without LU.

In order to assess the quality of continuous media transferred over the network, we transmitted an audio-video stream along a route selected by each routing protocol. We employed application-level QoS parameters such as the MU loss ratio and MU delay.

\section{A. Experiment I}

\section{1) Experimental System}

The experimental system is composed of five laptop PCs (IBM ThinkPad X31, Red Hat Linux 9) each equipped with an IEEE 802.11b PC card (Planex GW-NS11H). In this experiment, the wireless PC card changes the data rate automatically. As shown in Figure 4, the PCs were arranged on the same floor of a building. Figure 4 displays two cases of
Table 1: Specifications of Audio and Video.

\begin{tabular}{|c|c|c|}
\hline & Audio & Video \\
\hline Coding scheme & G.711 $\mu$-law & MPEG1 \\
\hline Image size & - & $320 \times 240$ pixels \\
\hline Picture pattern & - & I \\
\hline Playing time & \multicolumn{2}{|c|}{$180 \mathrm{~s}$} \\
\hline Average MU rate & $8 \mathrm{MU} / \mathrm{s}$ & $20 \mathrm{MU} / \mathrm{s}$ \\
\hline Average bit rate & $64 \mathrm{kbps}$ & $800 \mathrm{kbps}$ \\
\hline
\end{tabular}

the node arrangement: case 1 is represented by the white nodes, and case 2 the black nodes. In Figure 4, node 1 is the source, and node 5 is the destination. The objective of the two cases of the node arrangement is to examine the effect of the distances between the nodes on the QoS.

The device driver software for the wireless PC card is "HostAP" [15], which is modified so that it can measure the link quality. Open source software "Olsrd" [16] is used for OLSR. The software for LQHR is a modified version of the Olsrd in which the QM module and the RS module functions are embedded.

\section{2) Conditions of the Experiment}

Audio-video streams are transmitted from node 1 to node 5 . Table 1 shows the specifications of the audio and video. As mentioned earlier, an audio packet consisting of a constant number of audio samples is defined as an audio MU, and a video frame as a video MU.

In the QM module, each node sends five probe packets in a measurement at each of the four data rates which are specified in IEEE 802.11b [3]: 1, 2, 5.5 and 11 Mbps. That is to say, 20 probe packets are sent in total; they are sent at intervals of five seconds, and the source node also selects an up-to-date route at intervals of five seconds. The threshold $T h_{S N R}$ for SNR is set to be $20 \mathrm{~dB}$. A probe packet is a UDP datagram whose payload is 72 bytes. An RQReq message is a UDP datagram of 20 bytes, and the payload of the RQReq message is increased by four bytes each time it travels one hop. In experiment I, only SNR of received packets is used as the link quality.

\section{3) QoS Parameters}

In order to investigate the quality of a selected route, we employ the MU loss ratio and MU delay [17] as QoS parameters for application-level QoS assessment of audio and video transmission. The MU loss ratio is defined as the ratio of the number of MUs which are lost somewhere to the total number of MUs transmitted. The MU delay is defined as the time in seconds from the moment an MU is generated at the source until the instant the MU is output at the destination.

\section{B. Experiment II}

\section{1) Experimental System}

The experimental system is composed of six laptop PCs each equipped with an IEEE 802.11b PC card. The PCs were arranged on the same floor of a building as shown in Figure 5. 


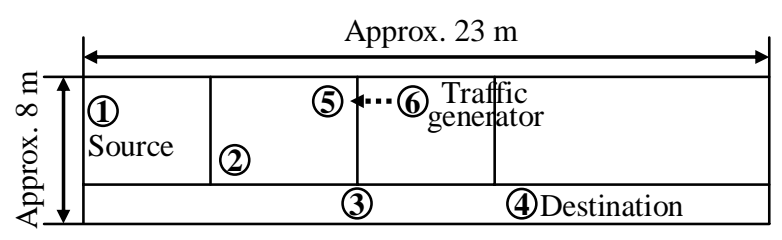

Figure 5: Arrangement of PCs in Experiment II.

Node 1 is the source, and node 4 is the destination. Node 6 generates a traffic flow and sends it to node 5 so as to make node 5 congested. The nodes are arranged so that SNR of a received packet sent from node 1 to node 2 , that from node 2 to node 3 , and that from node 3 to node 4 can be a value between 30 and $40 \mathrm{~dB}$, while the SNR in the cases of node 1 to node 5 and node 5 to node 4 can be between 20 and $30 \mathrm{~dB}$. The purpose of the node arrangement in experiment II is to make a difference in route quality between route "1-2-3-4" and route " $1-5-4$ ". The rest of the system configurations are the same as those in experiment I.

\section{2) Conditions of the Experiment}

Audio-video streams are transmitted from node 1 to node 4. The specifications of the audio and the video are the same as those in Table 1 except for the playing time, which is 60 seconds in experiment II. As a background traffic flow, an IP datagram of 1500 bytes is transmitted from node 6 to node 5 at regular intervals. Changing the transmission intervals adjusts the amount of traffic flow per unit time.

In experiment II, both SNR of received packets and link utilization level, which is defined by Eq. (1), are employed as the components of the link quality vector. The link utilization level is classified into three levels: low (1 through 20), middle (21 through 40), and high (more than 40). The traffic flow is adjusted so that the link utilization level at node 5 can be 0 , 15, 30 and 45, respectively. The rest of the experiment conditions are the same as those in experiment I.

\section{3) QoS Parameters}

To examine the effect of the network load on the temporal structure of the transmitted audio-video streams, we employ the MU delay and the coefficient of variation of MU output interval [17] as application-level QoS parameters. The coefficient of variation of MU output interval is defined as the ratio of the standard deviation of MU output interval to its mean value. This parameter represents the smoothness of output of a media stream.

\section{EXPERIMENTAL RESULtS}

In this section, we present the experimental results and discuss the effectiveness of LQHR in each of the two experiments: experiment I and experiment II.

\section{A. Experiment I}

In this section, we discuss the effectiveness of LQHR in comparison with the standard OLSR under the condition that there exists no other traffic flow in the network.

We first observed that LQHR always selected route "1-2-3$4-5$ ” in case 1 in Figure 4 (the group of white nodes) and

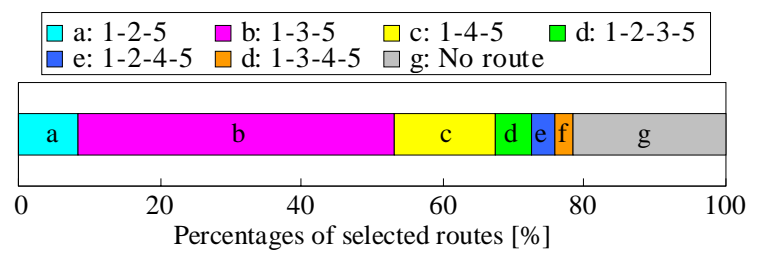

(a) Case 1

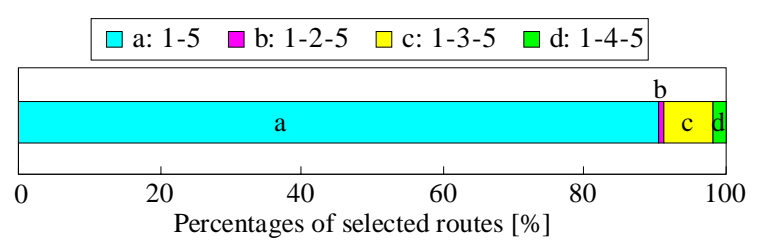

(a) Case 2

Figure 6: Percentages of routes selected by the standard OLSR.

route " $1-3-5$ " in case 2 (the group of black nodes). A sequence of "1-3-5", for example, denotes a two-hop route which consists of source node 1 , intermediate node 3 and destination node 5.

Regarding the standard OLSR, we show percentages of routes selected in cases 1 and 2 in Figure 6. In case 1, we see that two-hop route " $1-3-5$ " was selected by the standard OLSR most frequently; we regard this route as the representative one selected by the standard OLSR in case 1 . Recall that LQHR in case 1 always selected four-hop route "1-2-3-4-5", which is two hops longer than representative route " $1-3-5$ " for the standard OLSR; this is because SNR on link " $1-3$ " and that of link " $3-5$ " were smaller than the threshold $T h_{S N R}$. In case 2, one-hop route " $1-5$ " was selected by the standard OLSR with the percentage of nearly $90 \%$, since each node was located closely to one another as compared with case 1 ; we regard this route as the representative one selected by the standard OLSR in case 2. Note that two-hop route " $1-3-5$ ", which was always selected by LQHR, is not the maximum hop count route. This is because route " $1-3-5$ " has the minimum hop count to the destination among the routes which have SNR higher than the threshold $T h_{S N R}$.

In both cases, LQHR selected the routes whose hop count is more than that of the representative ones for the standard OLSR, since LQHR selected routes based on both hop count and SNR, whereas the standard OLSR selected shortest routes.

Figure 7 shows the MU loss ratio which was measured in every second when the nodes were arranged as case 2 of Figure 4. Figures 7-(a) and 7-(b) represent the MU loss ratio of audio and that of video, respectively; the MU loss ratio of the standard OLSR and that of LQHR are plotted by circle and diamond symbols, respectively. In both audio and video, we observe that MU loss occurs frequently in the standard OLSR, whereas it hardly happens in LQHR. In the standard OLSR, a data packet transmitted is likely to be corrupted since a route which is selected with the minimum hop count metric is unreliable. Moreover, when a routing control packet such as a HELLO message is lost, a route is considered broken, and then a new route is selected; thus, we can see successive packet loss around 30 second in Figure 7. On the other hand, 


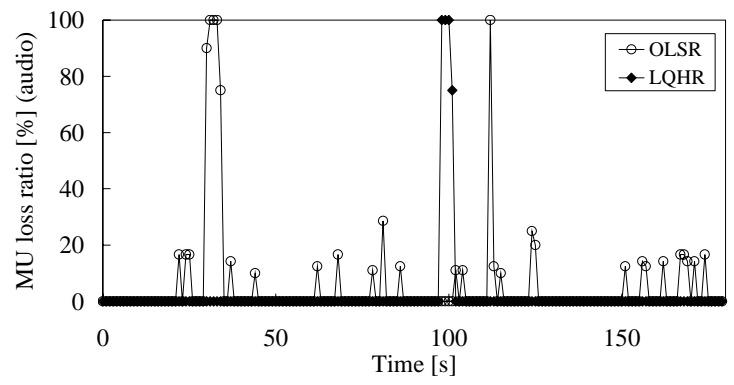

(a) Audio

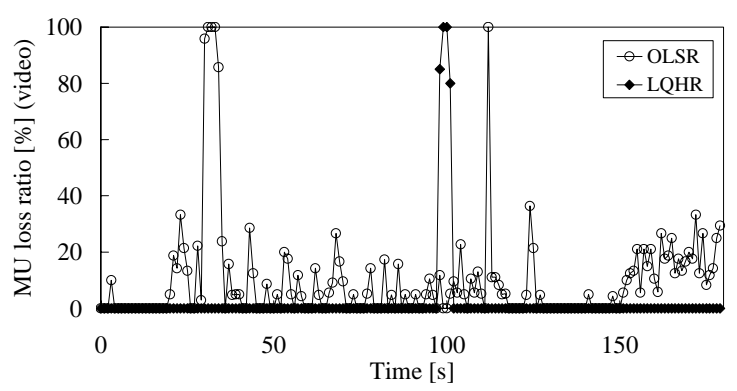

(b) Video

Figure 7: MU loss ratio as a function of time for audio and video in case 2.

the route in LQHR is stable, and hence packet loss scarcely occurs because a route is selected on the basis of SNR.

Figure 8 indicates the MU delay which was measured and averaged in every second when the nodes were arranged as case 2 of Figure 4. Figures 8-(a) and 8-(b) represent the MU delay of audio and that of video, respectively. In Figure 8, circle and diamond symbols denote the MU delay of the standard OLSR and that of LQHR, respectively. When the MU loss ratio is 100 [\%], i.e., when no MU reached the destination for the period of time, the MU delay is regarded as infinite and is not plotted in Figure 8. We see that the MU delay often increases in the standard OLSR, whereas it is almost constant in LQHR. When a packet is lost on the way to a receiver node, an ACK packet is not returned from the node, and then the sender node schedules retransmission of the packet. Because an MU is divided into a certain number of packets, the MU cannot be assembled even if one of the packets which comprise the MU is lost. This is why the retransmission due to packet loss increases the MU delay. In the standard OLSR, the MU loss occurs frequently as illustrated in Figure 7; thus, the MU delay increases significantly as compared with the case of LQHR.

\section{B. Experiment II}

In this section, we examine the effectiveness of LQHR under the condition that there is another traffic flow in the network. We employed two types of LQHR in experiment II: LQHR without LU and LQHR with LU. LQHR with LU is identical to the proposed LQHR. We compare LQHR with LU to the standard OLSR and LQHR without LU.

Table 2 shows the routes which were selected by the three routing protocols for four values of the link utilization level at node 5 , namely, $0,15,30$, and 45 . Note that node 5 receives

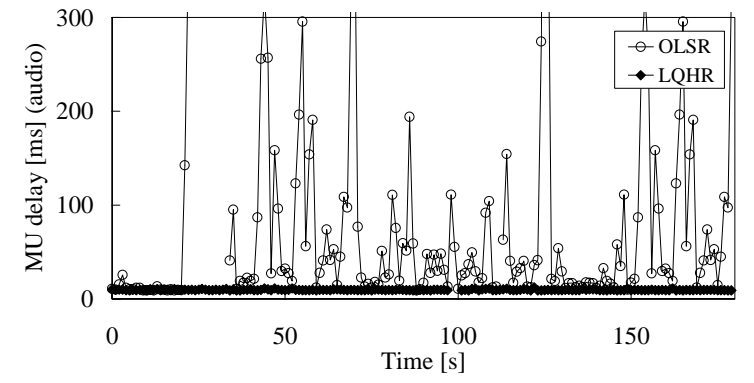

(a) Audio

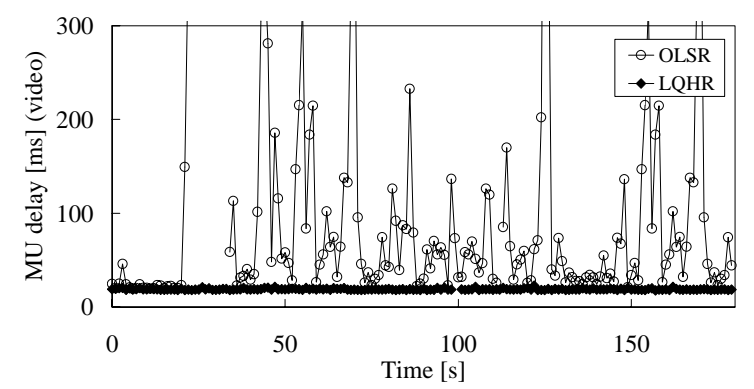

(b) Video

Figure 8: MU delay as a function of time for audio and video in case 2.

Table 2: Routes selected for four values of the link utilization level.

\begin{tabular}{|c|c|c|c|}
\hline $\begin{array}{c}\text { Link utilization } \\
\text { level at node 5 }\end{array}$ & OLSR & $\begin{array}{c}\text { LQHR } \\
\text { without LU }\end{array}$ & $\begin{array}{c}\text { LQHR } \\
\text { with LU }\end{array}$ \\
\hline 0 & $1-4$ & $1-5-4$ & $1-5-4$ \\
\hline 15 & $1-4$ & $1-5-4$ & $1-5-4$ \\
\hline 30 & $1-4$ & $1-5-4$ & $1-5-4$ \\
\hline 45 & $1-4$ & $1-5-4$ & $1-2-3-4$ \\
\hline
\end{tabular}

the interference traffic. In Table 2, we see that the standard OLSR always selected one-hop route " $1-4$ " at every link utilization level. On the other hand, when the link utilization level at node 5 is equal to or less than 30, both types of LQHR selected two-hop route " $1-5-4$ ", which we found from the experimental result the shortest route among those whose SNR in the route quality is higher than the threshold $T h_{S N R}$. When the link utilization level at node 5 is 45 , which is the high utilization level, LQHR with LU selected three-hop route "12-3-4" to avoid node 5, which was overloaded. On the other hand, LQHR without LU selected the two-hop route "1-5-4" even in this case.

Figure 9 and Figure 10 show the MU delay and the coefficient of variation of MU output interval, respectively, versus link utilization level. They were measured ten times and were averaged. In Figures 9 and 10, we observe that both types of LQHR indicate the significant improvement upon the standard OLSR in both MU delay and the coefficient of variation of MU output interval, especially when the link utilization level at node 5 is equal to or less than 30 . This is because both types of LQHR selected the routes which are one hop longer but more stable than the routes selected by the standard OLSR by referring to the SNR of each link. When the link 


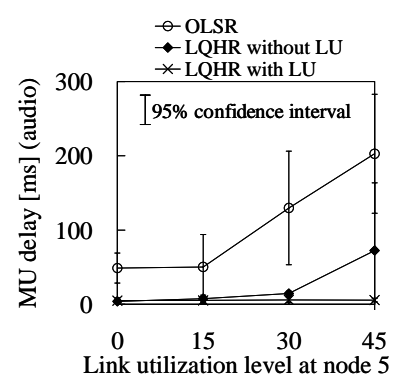

(a) Audio

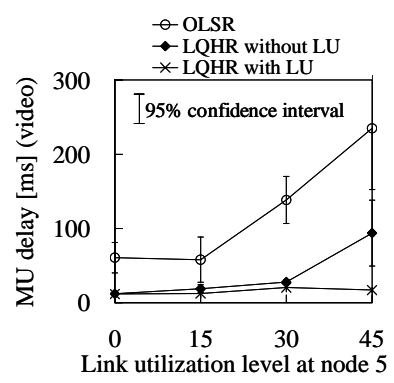

(b) Video

Figure 9: MU delay versus link utilization level for audio and video.

utilization level at node 5 is 45 , we notice that LQHR with LU achieves better quality than LQHR without LU. This means that LQHR with LU can find a suitable route by avoiding an overloaded node, i.e., node 5, though the hop count of the selected route becomes larger than that of the route selected by LQHR without LU. The route of LQHR with LU is two-hop longer than that of the standard OLSR; however, the longer route in this case outperforms the shortest route in MU delay. This suggests that a difference of a few hops between routes does not necessarily have a significant effect on MU delay; a reliable and less congested route contributes to the achievement of high quality audio-video transmission.

\section{CONCLUSIONS}

We have proposed LQHR, which is a combined protocol of proactive and reactive routing. In order to assess the effectiveness of LQHR, we have implemented LQHR on an experimental network and evaluated the audio-video transmission in terms of application-level QoS.

Our experimental results showed that LQHR can provide a better quality route in comparison to the standard OLSR since LQHR selects a route based on not only the minimum hop count metric but also the SNR between adjacent nodes and the link utilization of each node. Although the number of hops of a route selected by LQHR is larger than that of the shortest one, the LQHR route is reliable and less congested; it can provide lower transmission delay and smaller loss ratios of the audio-video streams.

Because LQHR utilizes SNR as one of routing metrics, its performance should be compared to that of the existing routing algorithms which also consider the link quality, such as FSR-SS and FSR-MS. In the experiment, we set the probe packet interval to 5 seconds, which was decided through a preparatory experiment. Although LQHR is designed not to introduce a heavy traffic load of control packets into the network, the evaluation of the influence of such probe packets on QoS is also one of our future studies.

\section{REFERENCES}

[1] Mobile Ad-hoc Networks (manet), http://www.ietf.org/html.charters/manet-charter.html.

[2] IEEE Std. 802.11, "Wireless LAN medium access control (MAC) and physical layer (PHY) specifications,” 1999.

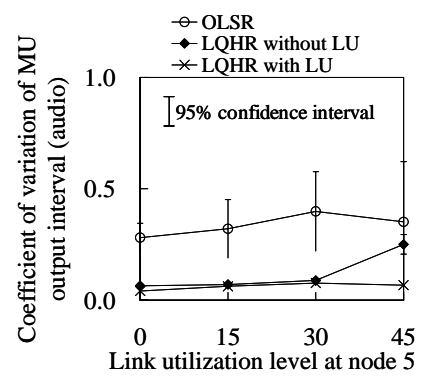

(a) Audio

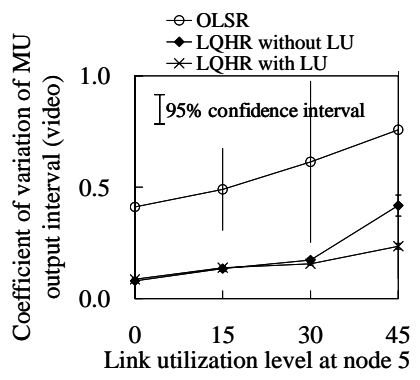

(b) Video
Figure 10: Coefficient of variation of MU output interval versus link utilization level for audio and video.

[3] IEEE Std. 802.11b, "Supplement to part 11: wireless LAN medium access control (MAC) and physical layer (PHY) specifications: higherspeed physical layer extension in the 2.4 GHz band,” Jan. 1999.

[4] Y. Owada, T. Suda, J. Prak and K. Mase, "Implementation of link stability-aware AODV and performance evaluation,” (in Japanese) Technical Report of IEICE Radio Communication Systems, RCS2004-7, pp. 3539, Apr. 2004.

[5] H. Lundgre, E. Nordstrom and C. Tschudin, "Coping with communication gray zones in IEEE 802.11b based ad hoc networks," in Proc. of the 5th ACM International Workshop on Wireless Mobile Multimedia, pp. 49-55, Sept. 2002.

[6] S. Itaya, J. Hasegawa, A. Hasegawa and P. Davis, “Achieving stable operation of ad hoc wireless networks with neighbour pre-selection and synchronous route updates,” in Proc. of the IEEE Conference on Local Computer Networks 30th Anniversary, pp. 697-702, Nov. 2005.

[7] B. Awerbuch, D. Holmer and H. Rubens, “ High throughput route selection in multi-rate ad hoc wireless networks,” in Proc. of 1st Working Conference on Wireless On-demand Network Systems, pp. 251-268, Jan. 2004.

[8] D. S. J. D. Couto, D. Aguayo, J. Bicket and R. Morris, "A highthroughput path metric for multi-hop wireless routing," in Proc. of the 9th annual International Conference on Mobile Computing and Networking, pp. 134-146, Sept. 2003.

[9] R. Dube, C. D. Rais, K.-Y. Wang and S. K. Tripathi "Signal stability based adaptive routing (SSA) for ad-hoc mobile networks,” IEEE Personal Communication, vol. 4, no. 1, pp. 36-45, Feb. 1997.

[10] S. Itaya, J. Hasegawa, M. Kosuga, T. Shimada and P. Davis, "Improving the stability of ad hoc wireless communications in an office environment with fluctuating radio conditions,” (in Japanese) IEICE Society Conference, BS-7-8, Sept. 2004.

[11] M. Watanabe, S. Tang and S. Tanaka, "Experimental result of ad hoc routing protocol due to the received signal strength in the office environment,” (in Japanese) IEICE Society Conference, B-5-125, Sept. 2004.

[12] T. Clausen and P. Jacquet, "Optimized link state routing protocol (OLSR),” RFC 3626, Oct. 2003.

[13] S.-J. Lee and M. Gerla, "Dynamic load-aware routing in ad hoc networks,” in Proc. of IEEE International Conference on Communications, pp. 3206-3210, Jun. 2001.

[14] S. Takahashi, J. Hakoda, H. Uehara and M. Yokoyama, "A load balanced routing scheme for mobile ad hoc networks,” in Proc. of International Symposium on Information Theory and its Applications, pp. 445450, Oct. 2004.

[15] Host AP driver for intersil Prism2/2.5/3, hostapd, and WPA Supplicant, http://hostap.epitest.fi.

[16] olsr.org, http://www.olsr.org.

[17] S. Tasaka and Y. Ishibashi, "Mutually compensatory property of multimedia QoS,” in Conf. Rec. of IEEE International Conference on Communications, pp. 1105-1111, Apr. 2002. 